\title{
The Influence of Self-Awareness, Self-Acceptance, and Empathy on Interpersonal Competence in Nursing Students
}

\author{
Sunghee Lee ${ }^{1}$ and Yeojeong $\mathrm{Nam}^{2}$ \\ ${ }^{1}$ Professor, College of Nursing, The Research Institute of Nursing, Kyungpook \\ National University, 680, Gukchaebosang-ro, Jung-gu, Daegu 41944, KOREA \\ ${ }^{2}$ Graduate Student, Graduate School of Nursing, Kyungpook National University, 680, \\ Gukchaebosang-ro, Jung-gu, Daegu 41944, KOREA \\ ${ }^{1}$ leesh@knu.ac.kr, ${ }^{2}$ Corresponding Author: namyj@knu.ac.kr
}

\begin{abstract}
The purpose of this study was to examine factors influencing the interpersonal competence of nursing students. The data used in this study were collected between 1st August 2016 and 31 st August 2016 from 168 students who were majoring in nursing in a four-year university at Daegu, South Korea. Data were analyzed using measures of frequency, percentage, mean, and standard deviation, in addition to a t-test, One-way ANOVA, Tukey test, Pearson's correlation coefficient, and multiple regression analysis using IBM SPSS Statistics version 21.0. The factors that influence the interpersonal competence of nursing students were age $(\beta=.194, p=.006)$, self-awareness $(\beta=.406, p<.001)$, self-acceptance $(\beta=.278, p<.001)$, and empathy $(\beta=.164, p=.015)$. These variables explained $34.5 \%$ of the variance in interpersonal competence scores. The results show that age, self-awareness, self-acceptance, and empathy influenced interpersonal competence. Based on these results, intervention programmes that can enhance the influencing factors of interpersonal competence in nursing students should be developed, and their effectiveness should be investigated.
\end{abstract}

Keywords: Self-Awareness, Self-Acceptance, Empathy, Interpersonal, Competence

\section{Introduction}

\subsection{Study rationale}

Individuals with high interpersonal competence have greater psychological well-being [1], high self-esteem [2], which allows for ideal growth and development [3] as well as successful adjustment in social organizations [4]. In contrast, individuals with low interpersonal competence experience negative emotions, such as anxiety, depression, low self-esteem, and aggression [5], and ultimately suffer from conflicts and maladjustments in society and various organisations [6].

In the same vein, whereas nurses with high interpersonal competence have high self-esteem [7] and perceive nursing work as positive [8], those with low self-esteem suffer from stress related to the organisational work environment due to declines in nursing performance [9], which eventually leads to turnover [10]. Interpersonal competence in nurses

Article history:

Received (September 03, 2016), Review Result (October 07, 2016), Accepted (November 11, 2016) 
may be cultivated through nursing education programmes and various extracurricular activities [11].

However, many recent studies of nursing students have reported that students experience anxiety and stress during clinical training due to their lack of interpersonal competence and even abuse other people physically, verbally, and emotionally [12][13]. Therefore, there is a great need for intervention measures to promote interpersonal competence among nursing students; this presupposes the systemic exploration of factors affecting nursing students' interpersonal competence.

This study seeks to elucidate the effects of self-awareness, self-acceptance, and empathy on nursing students' interpersonal competence, and provide the results as the basis for developing nursing intervention measures promoting interpersonal competence.

\subsection{Study objectives}

To investigate differences in interpersonal competence among nursing students according to their general characteristics; to identify the degrees of self-awareness, self-acceptance, empathy, and interpersonal competence of nursing students; to elucidate the relationships between nursing students' interpersonal competence, self-awareness, self-acceptance, and empathy; to confirm the influences of self-awareness, self-acceptance, and empathy on interpersonal competence of nursing students.

\section{Methods}

\subsection{Participants}

Participants in this study were 168 nursing students of K University in D city. Although 186 students initially participated in the study, 18 students with incomplete survey results were excluded, and 168 students were ultimately included in the data analysis. According to $\mathrm{G}^{*}$ power 3.1 [14], the minimum sample size required for multiple regression analysis with an effect size of .15, significance level of .05, and statistical power of .90, was 116 .

\subsection{Measurement tools}

\subsubsection{Interpersonal competence}

In order to measure interpersonal competence, we used the version of the Buhrmester et al. [15] Interpersonal Competence Questionnaire (ICQ) validated by Han and Lee [16]. This questionnaire consists of 31 questions, each measured on a five-point Likert scale ( $1=$ strongly disagree, $5=$ strongly agree). The ICQ was found to have good internal consistency in Han and Lee's study [16], with the authors reporting a Cronbach's $\alpha$ of .84. The Cronbach's $\alpha$ in the present study was 90 .

\subsubsection{Self-awareness}

Self-awareness was measured with a self-awareness scale originally developed by Fenigstein et al. [17] and later modified by Eun [18]. This scale consists of 20 questions, each measured on a five-point Likert scale ( $1=$ strongly disagree, $5=$ strongly agree). The scale was reported to have a Cronbach's $\alpha$ of .74 by Eun [18], and has a Cronbach's $\alpha$ of .70 in the present study. 


\subsubsection{Self-acceptance}

In order to measure self-acceptance, we used the version of Phillips' [19] Self-Acceptance Scale modified by Son [20]. This scale consists of 25 questions each measured on a five-point Likert scale (1=always, 5=never). The scale had a Cronbach's $\alpha$ of .84 in Phillips' [19] original study, and a Cronbach's $\alpha$ of .89 in the present study.

\subsubsection{Empathy}

Empathy was measured with the version of Davis' [21] Interpersonal Reactivity Index (IRI) modified by Park[22]. The index comprises two sub-scales: cognitive empathy and emotional empathy. There are a total of 28 questions, each of which is measured on a five-point Likert scale (1=strongly disagree, 5=strongly agree). Park [22] reported a Cronbach's $\alpha$ of .84 for the IRI; the Cronbach's $\alpha$ for the present study was .79.

\subsection{Data collection}

Data collection for this study was performed after obtaining consent from both the dean of a 4-year nursing school in D city and the appointed professor of a course at the school in August 2016. We obtained consent from all participants, and it took approximately 15 minutes to complete the survey questionnaire.

\subsection{Data analysis}

Data were analysed using IBM SPSS Statistics version 21.0. Participants' general characteristics were analysed, and each variable was analysed for frequency, percentage, mean, and standard deviation. Differences in interpersonal competence arising due to these general characteristics were analysed via t-tests and one-way ANOVA. Correlations between self-awareness, self-acceptance, empathy, and interpersonal competence were identified with Pearson's correlation coefficient, and the factors that influence interpersonal competence were analysed through multiple regression analysis.

\section{Results}

\subsection{General characteristics and their relationship with interpersonal competence}

The majority of participants, $90.5 \%$, were female, $42.3 \%$ were $20-21$ years of age, and $28.0 \%$ were in their $4^{\text {th }}$ year. Students without previous clinical practice experience accounted for $52.4 \%$ of the sample. Most participants had one sibling (66.1\%), and $10.1 \%$ had three or more siblings. Among the subjects, $70.2 \%$ participated in one club activity, and $63.1 \%$ indicated that they had participated in voluntary work more than twice. Analysis of the differences in interpersonal competence scores based on general characteristics revealed a significant difference only for age; participants over 22 years of age had significantly higher interpersonal competence scores when compared to those who were 18-19 years of age $(\mathrm{F}=-3.310, p=.039)$.

\subsection{Degree of self-awareness, self-acceptance, empathy, and interpersonal competence}

The average self-awareness score was $68.36 \pm 5.97$, and the average for self-acceptance was $78.83 \pm 11.30$. The average score for empathy was $98.84 \pm 8.05$, and the average for interpersonal competence was 101.41 \pm 14.36 . 


\subsection{Relationships among self-awareness, self-acceptance, empathy, and interpersonal competence}

Self-awareness and self-acceptance showed significant positive correlations with interpersonal competence ( $\mathrm{r}=.491, p<.001$ and $\mathrm{r}=-.327, p<.001$, respectively). Furthermore, empathy also showed a significant positive correlation with interpersonal competence $(\mathrm{r}=-.240, p=.002)$.

\subsection{The influence of self-awareness, self-acceptance, and empathy on interpersonal competence}

In order to identify the factors that influence interpersonal competence, we set the age of participants as a dummy variable and performed a regression analysis on the following variables, which had significant positive correlations with interpersonal competence: self-awareness, self-acceptance, and empathy.

The Durbin-Watson value was 1.890 , which showed that there was no autocorrelation. Tolerance, which ranged between .818 and .933 , was well over 0.1 . The Variance inflation factor (VIF), which ranged between 1.072 and 1.223, was smaller than 10. These results indicate that all variables were free from multicollinearity. Stepwise multiple regression analysis results identified age $(\beta=.194, \quad p=.006)$, self-awareness $(\beta=.406, \quad p<.001)$, self-acceptance $(\beta=.278, p<.001)$, and empathy $(\beta=.164, p=.015)$ as factors with influence on interpersonal competence. Being older than 22 as well as higher self-awareness, self-acceptance, and empathy scores corresponded to higher interpersonal competence. These four variables explained $34.5 \%$ of the variance in interpersonal competence [Table 1].

Table 1. Factors influencing interpersonal competence among participants $(\mathrm{N}=168)$

\begin{tabular}{|c|c|c|c|c|c|c|c|}
\hline Variables & $\mathrm{B}$ & $B$ & $\mathrm{t}$ & $p$ & $\mathrm{R}^{2}$ & $\operatorname{Adj}^{2}$ & $\mathrm{~F}(p)$ \\
\hline (Constant) & -24.276 & - & -1.625 & .106 & .364 & .345 & $\begin{array}{c}18.573 \\
(<.001)\end{array}$ \\
\hline$\geq 22^{\dagger}$ & 7.060 & .194 & 2.803 & .006 & - & - & - \\
\hline Self-awareness & .978 & .406 & 6.090 & $<.001$ & - & - & - \\
\hline Self-acceptance & .354 & .278 & 4.287 & $<.001$ & - & - & - \\
\hline Empathy & .293 & .164 & 2.452 & .015 & - & - & - \\
\hline
\end{tabular}

$广$ Reference: 18-19 years of age

\section{Conclusion and suggestions}

This descriptive study sought to provide the basic data required for the development of a nursing education programme promoting interpersonal competence, by elucidating the influence of self-awareness, self-acceptance, and empathy on the interpersonal competence of student nurses. The results show that age, self-awareness, self-acceptance, and empathy all exert influence on nursing students' interpersonal competence. Based on the findings of this study, we suggest the need to develop and implement a nursing education programme that promotes self-awareness, self-acceptance, and empathy to ultimately promote interpersonal competence in student nurses. 


\section{References}

[1] C. D. Ryff and C. L. M. Keyes, "The structure of psychological well-being revisited," Journal of Personal and Social Psychology, vol.69, no.4, pp.719-727, (1995) DOI:10.1037/0022-3514.69.4.719

[2] S. M. Bae, "Validation of the interpersonal competence questionnaire in korean adults," Korea Journal of Counselimg, vol.16, no.3, pp.51, (2015)

[3] M. S. Lee and K. M. Chae, "The mediating effects of emotion regulation strategies on the relationship between self-esteem and interpersonal relationship abilities," Cognitive Behavioural Therapy Korea, vol.12, no.1, pp.63-75, (2012)

[4] C. Segrin and M. Taylor, "Positive interpersonal relationships mediate the association between social skills and psychological well-being," Personal and Individual Differences, vol.43, no.4, pp.637-646, (2007) DOI:10.1016/j.paid.2007.01.017

[5] M. R. Leary and R. F. Baumeister, "The nature and function of self-esteem: Sociometer theory," Academic Press Inc., San Diego, vol.32, pp.1-62, (2000) DOI:10.1016/S0065-2601(00)80003-9

[6] J. Gaines and J. M. Jermier, "Emotional exhaustion in a high stress organization," Academy of Management Journal, vol.26, no.4, pp.567, (1983) DOI:10.5465/255907

[7] G. J. Olthuis, C. J. W. Leget, and W. J. M. Dekkers, "Why hospice nurses need high self-esteem," Nursing Ethics, vol.14, no.1, pp.62-71, (2007) DOI:10.1177/0969733007071359

[8] H. S. Lee and J. K. Kim, "Relationship among communication competence, communication types, and organizational commitment in hospital nurses," Journal of Korean Academy for Nursing Administration, vol.16, no.4, pp.488-496, (2010)

[9] M. P. Leiter and C. Maslach, "The impact of interpersonal environment on burnout and organizational commitment: Summary," Journal of Behavioural Organ, vol.9, no.4, pp.297, (1988) DOI:10.1002/job.4030090402

[10] Y. J. Lee and K. Kim, "Experiences of nurse turnover,” Journal of Korean Academy of Nursing, vol.38, no.2, pp.248-257, (2008) DOI:10.4040/jkan.2008.38.2.248

[11] P. Morrison and P. Burnard, 'Students' and trained nurses' perceptions of their own interpersonal skills: A report and comparison," Journal of Advance Nursing, vol.14, no.4, pp.321-329, (1989) DOI:10.1111/j.1365-2648.1989.tb03419.x

[12] Y. Choi and M. Won, "A pilot study on effects of a group program using recreational therapy to improve interpersonal relationships for undergraduate nursing students," Archives of Psychiatric Nursing, vol.27, no.1, pp.54-55, (2013) DOI:10.1016/j.apnu.2012.08.002

[13] J. Longo, "Horizontal violence among nursing students," Archives of Psychiatric Nursing, vol.21, no.3, pp.177-178, (2007) DOI:10.1016/j.apnu.2007.02.005

[14] F. Faul and E. Erdfelder, A. Buchner and A.-G. Lang, "Statistical power analyses using g*power 3.1: tests for correlation and regression analyses," Behavioural Research Method, vol.41, pp.1149-1160, (2009) DOI:10.3758/BRM.41.4.1149

[15] D. Buhrmester, W. Furman, M. T. Wittenberg, and H. T. Reis, "Five domains of interpersonal competence in peer relationships," Journal of Personal and Social Psychology, vol.55, no.6, pp.991-1008, (1988) DOI:10.1037/0022-3514.55.6.991

[16] N. R. Han and D. G. Lee, "Validation of the Korean version of the interpersonal competence questionnaire in Korean college students," Korean Journal of Counselling and Psychotherapy, vol.22, no.1, pp.137-156, (2010)

[17] A. Fenigstein, M. F. Scheier, and A. H. Buss, "Public and private self consciousness: Assessment and theory," Journal of Consulting and Clinical Psychology, vol.43, no.4, pp.522-527, (1975) DOI: $10.1037 / \mathrm{h} 0076760$

[18] H. G. Eun and Adolescents, "Self-awareness, other-awareness, interpersonal skills and the satisfaction of interpersonal relationship: The difference among the grades and between the sexes," Korean Journal of 
The Influence of Self-Awareness, Self-Acceptance, and Empathy on Interpersonal Competence in Nursing Students

Youth Counselling, vol.9, pp.136-157, (2001)

[19] E. L. Phillips, "Attitudes toward self and others: A brief questionnaire report," Journal of Consulting Psychology, vol.15, no.1, pp.79-81, (1951) DOI:10.1037/h0054271

[20] I. H. Son, "The effects of small group study on academic achievements, and self-acceptance and other acceptance," Graduate School of Education Dong-A University, (1987)

[21] M. H. Davis, "Measuring individual differences in empathy: Evidence for a multidimensional approach," Journal of Personal and Social Psychology, vol.44, no.1, pp.113-126, (1983) DOI:10.1037/0022-3514.44.1.113

[22] S. H. Park, "Empathy and prosocial behaviour," Moonumsa, (1997) 